\title{
Modified Nanodiamonds as a New Carrier for Developing Reusable Enzymatic Test-Systems for Determination of Physiologically Important Substances
}

\author{
Nikita Ronzhin ${ }^{1 *}$, Alexey Baron ${ }^{1,2}$, Alexey Puzyr ${ }^{1}$, Irina Baron ${ }^{3}$, Andrey Burov ${ }^{1,4}$, Vladimir Bondar $^{1}$ \\ ${ }^{1}$ Institute of Biophysics, Federal Research Center "Krasnoyarsk Science Center of Siberian Branch of Russian \\ Academy of Sciences", Krasnoyarsk, Russia \\ ${ }^{2}$ Siberian Federal University, Krasnoyarsk, Russia \\ ${ }^{3}$ Voino-Yasenetsky State Medical University, Krasnoyarsk, Russia \\ ${ }^{4}$ Institute of Computational Technologies, Siberian Branch of Russian Academy of Sciences, Krasnoyarsk, Russia \\ Email: roniol@mail.ru
}

\begin{abstract}
The study demonstrates the application of detonation nanodiamonds in designing testsystems for determination of physiologically important blood substances. One (urease), two (glucose oxidase and peroxidase) and three (cholesterol esterase, cholesterol oxidase and peroxide) enzymes have been covalently immobilized onto the surface of modified nanodiamonds (MND) to develop testsystems for biochemical detection of urea, glucose, and total cholesterol, respectively. In all cases, the immobilized enzymes exhibit functional activity and catalyze the formation of a colored product in the presence of the analyte. It has been found that the obtained MND-enzyme(s) complexes effectively function in deionized water and different buffers providing a linear output of the final product over a wide range of temperatures, $\mathrm{pH}$ and analyte concentrations. The model biosensors were found to be suitable for repeated use and retain most of their activity after storage at $4{ }^{\circ} \mathrm{C}$ in deionized water for two months. Practical applicability of the glucose and total cholesterol biosensors is demonstrated in human serum samples.
\end{abstract}

Keywords: Biosensor, nanodiamonds, enzyme immobilization, glucose, cholesterol, urea.

\section{Introduction}

Continuous and reliable monitoring of key metabolites such as glucose, urea and cholesterol in blood serum samples is of paramount importance in diagnosis and prognosis for a number of diseases. Keeping the blood glucose levels within a specified range is particularly important in diabetes management. The increase in urea concentration in blood is associated with rental dysfunction. There is a strong correlation between a high blood serum cholesterol level and increased risk of developing coronary heart disease and liver failure, while the falling cholesterol level may indicate a disease process such as hyperthyroidism or cancer. Therefore, the development of high sensitive, reproducible, low-cost and easy to use sensors for clinic analysis has been the subject of concern for decades.

In recent years, a great number of enzyme-based analytical systems have been developed for qualitative and quantitative detection of various chemical and biological substances [1-6]. The demand for such systems is increasing due to their high selectivity and sensitivity, relative simplicity and rapidity of analysis. Enzymatic biosensors have been employed across a diverse range of applications, including environmental and agricultural monitoring, healthcare, food and water control [7-11].

Applications of nanomaterials have extended into the field of biomedical sensing expanding the arsenal of currently employed techniques with recent studies that demonstrate their great potential in improving the sensitivity and performance of biosensors [12-15]. There has been a steadily growing interest in using different allotropes of nanocarbon (fullerenes, graphene, nanotubes and nanodiamonds) as carriers and sensing elements for the design of biosensors [16-18]. Consequently, detonation nanodiamonds $[19,20]$ are well explored for health related issues due to their chemical stability, large specific area, facile surface tailoring for specific applications, high adsorption capacity and non-toxicity [21-24]. Along with a high colloidal stability in dispersion media, these properties of modified 
nanodiamonds (MND) provide for their potential use as a basis for a new class of bioanalytical devices for targeting and quantifying analytes of interest [25-28].

In this paper, we demonstrate the applicability of MND for constructing reusable diagnostic systems for the biochemical detection of glucose, total cholesterol and urea in blood serum.

\section{$2 \quad$ Materials and Methods}

\subsection{Materials and Reagents}

Nanodiamond powder synthesized by explosive detonation with an average cluster size of $50 \mathrm{~nm}$ were acquired from Real-Dzerzhinsk Ltd. (Russia) and used in this study as the starting material. In order to remove impurities and enhanced colloidal stability, the nanodiamonds were treated using a technique described earlier [24, 28]. Aqueous suspensions at concentration of $10 \mathrm{mg} / \mathrm{mL}$ were prepared by dispersing the modified nanodiamond (MND) powder in deionized (DI) water (Milli-Q system, Millipore USA).

Enzyme and analyte solutions supplied in commercial kits Cholesterol-Vital ("Vital Diagnostic", S.Petersburg, Russia), Glucose LS (ProDia International, Germany), and Novokarb ("Vector-Best", Russia) were used for the determination of total cholesterol, glucose, and urea in blood serum and plasma. Before experiments, to get rid of low molecular weight compounds the enzyme solutions were dialyzed against $10 \mathrm{mM}$ phosphate buffer (PH 7.0) by ultrafiltration through a $30 \mathrm{kDa}$ membrane (EMD Millipore Amicon, Darmstadt, Germany).

Phosphate, Bis-tris propane, tris, sodium acetate and ammonium acetate buffers ("Sigma", USA), 4aminoantipyrine (1-phenyl-2,3-dimethyl-4-aminopyrazolone) (4-AAP) and D-glucose ("Reahim", Russia), phenol ("Flucka", Germany), sodium hypochlorite and sodium hydroxide ("Vector-Best", Russia) were of analytical grade and employed without further purification. All solutions were prepared in DI water.

\section{$2.2 \quad$ Functionalization of MND}

The surface of MNDs was chemically activated with p-benzoquinone using a known technique [29, 30] that provides sites for the following immobilization of enzymes in relatively soft conditions. It is known [31] that in weak alkaline aqueous solutions hydroquinone is susceptible to oxidation forming pbenzoquinone. A phosphate buffer $(20 \mathrm{mM}, \mathrm{pH} 8.0)$ and $300 \mathrm{mg}$ of hydroquinone dissolved in $3 \mathrm{ml}$ of aqueous ethanol (20\%) were consequently added with stirring to an aliquot $(5 \mathrm{ml})$ of MND hydrosols (at final concentration of $1.0 \mathrm{wt} \%$ ) and the suspensions were incubated for $2 \mathrm{~h}$ at room temperature under stirring at $150 \mathrm{rpm}$ on a OS-10 Shaker (BIOSAN, Latvia). To remove unreacted components, MNDs were collected by centrifugation at $16000 \mathrm{~g}$ for $10 \mathrm{~min}$ at $10{ }^{\circ} \mathrm{C}$ and the sediment was washed with DI water followed by several cycles of twice washing with aqueous ethanol (20\%) and once with $1 \mathrm{M} \mathrm{NaCl}$, centrifugation and rinsing with water.

\subsection{Construction of MND-Enzyme(s) Complexes}

Three test-systems were constructed using identical procedures: 1) urease, 2) glucose oxidase and peroxidase, and 3) cholesterol esterase, cholesterol oxidase and peroxide enzymes have been covalently immobilized onto the surface of MNDs for biochemical detection of urea, glucose, and total cholesterol, respectively. Immobilization of the enzymes on the activated MNDs was carried out in $100 \mathrm{mM} \mathrm{Na-}$ bicarbonate buffer ( $\mathrm{pH}$ 8.0). A solution of the corresponding enzyme(s) was mixed with MND hydrosols and kept for 2 hours at room temperature under continual stirring (Shaker OS-10). Then the MNDenzyme complexes were collected by centrifugation (Centrifuge $5415 \mathrm{R}$ ) at $16000 \mathrm{~g}$ for $10 \mathrm{~min}$ at $10^{\circ} \mathrm{C}$. The precipitate was repeatedly washed with a $0.25 \mathrm{M} \mathrm{NaCl}$ solution to remove unbound and nonspecifically bound enzymes.

\subsection{Measure of Activity of MND-Enzyme(s) Complexes}

Activity of the immobilized enzymes in systems designed for detection of glucose and total cholesterol 
was assayed by colorimetric method using $\mathrm{H}_{2} \mathrm{O}_{2}$, 4-AAP and phenol involved in an oxidative coupling reaction [32]. Phenol and 4-AAP reacts in the presence of $\mathrm{H}_{2} \mathrm{O}_{2}$ and peroxidase resulting in formation of the colored product - quinoneimine dye. This reaction is commonly used in medical diagnostics for evaluation of physiologically important substances [33]. In a procedure for the enzymatic determination of glucose the colored product is formed by two sequential reactions (Fig. 1a), while three reactions are required for the determination of total cholesterol (Fig. 1b).
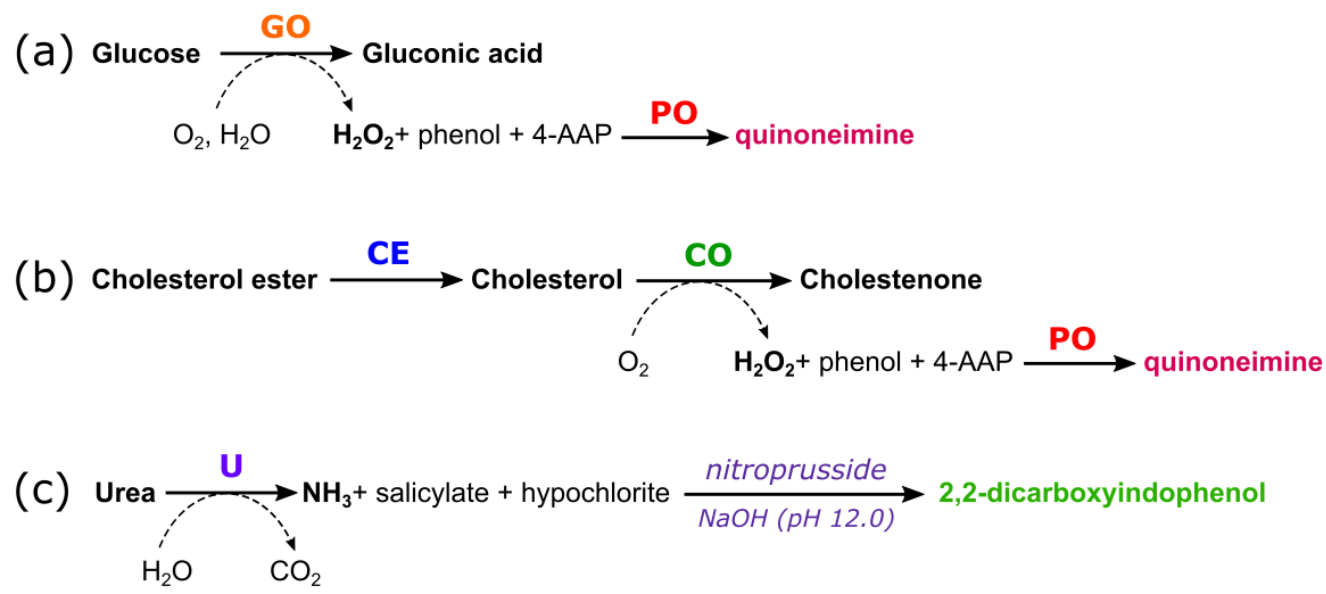

Figure 1. A schematic representation of glucose, cholesterol and urea assay methods. (a) glucose oxidaseperoxidase test for glucose determination, GO-glucose oxidase, PO-peroxidase. (b) cholesterol oxidase-peroxidase test for total cholesterol determination, CE-cholesterol esterase, CO-cholesterol oxidase, PO- peroxidase. (c) urease salicylate-hypochlorite test for urea determination, U-urease.

The assay $(1 \mathrm{ml})$ contained $0.56 \mathrm{mg} / \mathrm{ml}$ of phenol, $0.10 \mathrm{mg} / \mathrm{ml}$ of 4-AAP and $50 \mu \mathrm{l}$ of MND-enzymes suspension (at final concentration of $0.5 \mathrm{~g} / \mathrm{L}$ ). After addition of an analyte (cholesterol or glucose), the samples were vortexed for 3-5 s and incubated at room temperature. The analyte concentration, temperature, $\mathrm{pH}$ and incubation time were varied to study dependencies of colored product formation. The reaction mixture was centrifuged at $16000 \mathrm{~g}$ for $10 \mathrm{~min}$. The absorbance of the supernatants at 506 nm was measured by using a UV-1800 (Shimadzu, Japan) spectrophotometer.

For the urease activity assay, a salicylate-hypochlorite method [34,35] based on successive enzymatic and nonenzymatic reactions was used. First, urea is hydrolyzed by urease to form ammonia which at the nonenzymatic stage reacts under alkaline conditions with chemical components to form a colored product - 2,2-dicarboxyindophenol (Fig. 1c). The initial reaction mixture $(200 \mu \mathrm{L})$ contained $140 \mu \mathrm{L}$ of $10 \mathrm{mM}$ phosphate buffer $(\mathrm{pH} 7.0), 10 \mu \mathrm{L}$ of the urea standard (the analyte concentration of 0.25 $\mathrm{mmol} / \mathrm{L}$ ), and $50 \mathrm{\mu L}$ of the MNDs-urease suspension (the particle concentration of $2.5 \mathrm{~g} / \mathrm{L}$ ). After adding all components, the samples were mixed and incubated at room temperature for 5 min. After incubation, the MNDs-urease complex was collected by centrifugation at $16000 \mathrm{~g}$ for $10 \mathrm{~min}$. The obtained supernatants were collected and mixed with the chemical components to perform the nonenzymatic reaction. After mixing and incubation at room temperature for 5 min, the amount of the colored reaction product in the samples was determined spectrophotometrically by the optical density at 700 nm using a UV 1800 spectrophotometer (Shimadzu, Japan).

\section{$2.5 \quad$ Multiple Re-using of MND-Enzyme(s) Complexes}

Following the construction procedure described above, the test-systems for glucose and cholesterol detection were assessed for reproducibility in multiple use. For this purpose, MND-enzymes complexes were collected by centrifugation and the precipitates were washed twice with $20 \mathrm{mM}$ phosphate buffer (PH 7.0) to remove the reaction product. The washed MND-enzymes complexes were re-suspended in DI water, then analytes and ingredients required to start the reaction were again added.

In the case of multiple detection of urea level, the MND-urease complex was collected by centrifugation after the enzymatic stage. The supernatant was used for nonenzymatic reaction and 
analysis of the formed colored product. The pellet was washed twice with $20 \mathrm{mM}$ phosphate buffer ( $\mathrm{pH}$ 7.0) to remove the residual components and products of the enzymatic reaction, each time re-suspending the pellet in a freshly prepared buffer and collecting by centrifugation. The washed MND-urease complex was re-suspended in DI water and the enzymatic reaction was again activated by the analyte.

\subsection{Determination of Glucose and Cholesterol Concentration in Samples of Human}

\section{Blood Serum}

The analysis of glucose and cholesterol concentration with the developed test-systems was performed at room temperature on samples of human blood serum. An initial reaction mixture consisted of 0.56 $\mathrm{mg} / \mathrm{ml}$ of phenol, $0.10 \mathrm{mg} / \mathrm{ml}$ of 4-AAP and $50 \mathrm{pl}$ of either two- or three-enzymes-MND suspension for determination of glucose or cholesterol, correspondingly. Then a $50 \mathrm{pl}$ aliquot of blood serum was added to the reaction mixture and stirred for $5 \mathrm{sec}$ followed by incubation for $10 \mathrm{~min}$. After centrifugation to remove MND-enzymes complexes, the supernatant was used to quantify the analyte concentration from the optical density at $506 \mathrm{~nm}$. Calibration curves were obtained by assaying samples containing the colored product derived at various known concentrations of the analytes.

In parallel experiments, the concentration of glucose and cholesterol was determined with a Sapphire400 biochemical analyzer (Niigata Mechatronics, Japan). The Cholesterol-Vital (Vital Diagnostic, S.Petersburg, Russia) and Glucose LS (ProDia International, Germany) reagent kits were used to calibrate the analyzer following the producer instruction. The data obtained by different test methods were used for comparative analysis.

\section{$3 \quad$ Results and Discussion}

To construct test-systems for biochemical diagnostics, a method of covalent attachment of enzymes to MND activated with benzoquinone was applied (Fig. 2). It is known that in order to prevent denaturation of biopolymers (e.g. proteins) during their covalent immobilization on a carrier, its surface is pre-activated thus allowing them to bind biomolecules under relatively mild conditions [29-31]. As a result of reaction with the chemical activator, the reactive electrophilic groups developed on the carrier surface form covalent bonds with the nucleophilic groups (e.g. amino groups) of the biopolymer. A particular case of this reaction is the activation of a carrier containing surface- $\mathrm{OH}$ groups by benzoquinone and subsequent attachment of a protein to the activated carrier [29-31]. The method is widely used in the development of affinity sorbents to retain to a considerable extent the specific activity of immobilized proteins [31]. The abundance of hydroxyl groups on the MND surface has been shown earlier [27], therefore, in this study the enzymes were covalently immobilized onto the benzoquinone activated nanoparticles.

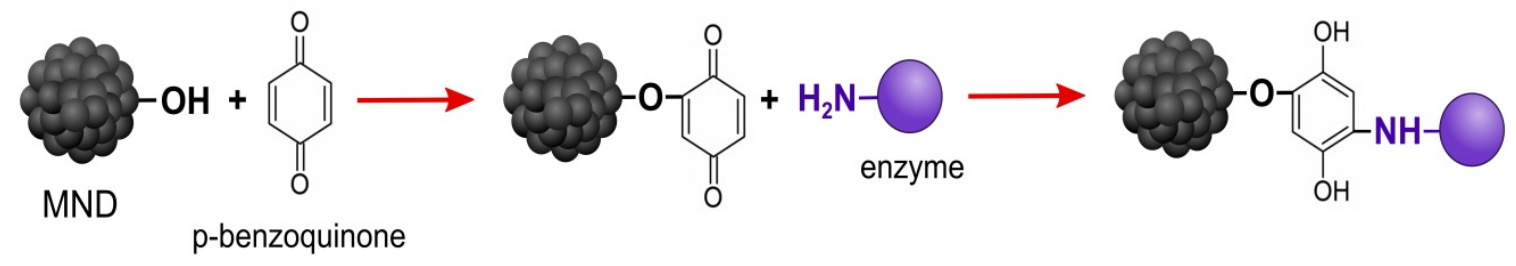

Figure 2. A scheme of functionalization of nanoparticle surface and covalent immobilization of enzymes onto MND.

A principle of functioning of model biosensors based on a MND-enzyme(s) complex is shown on the Fig. 3. A substance to be determined (the target analyte) is successively transformed into respective products $\mathrm{P}_{1} \rightarrow \mathrm{P}_{2} \rightarrow \ldots \rightarrow \mathrm{P}_{\mathrm{n}}$ by a chain of biochemical reactions catalyzed by enzymes $\mathrm{E}_{1} \rightarrow \mathrm{E}_{2} \rightarrow \ldots \rightarrow \mathrm{E}_{\mathrm{n}}$ immobilized on the nanoparticles. The final colored product $\left(\mathrm{P}_{\mathrm{n}}\right)$ is easily detected by spectral analysis. 

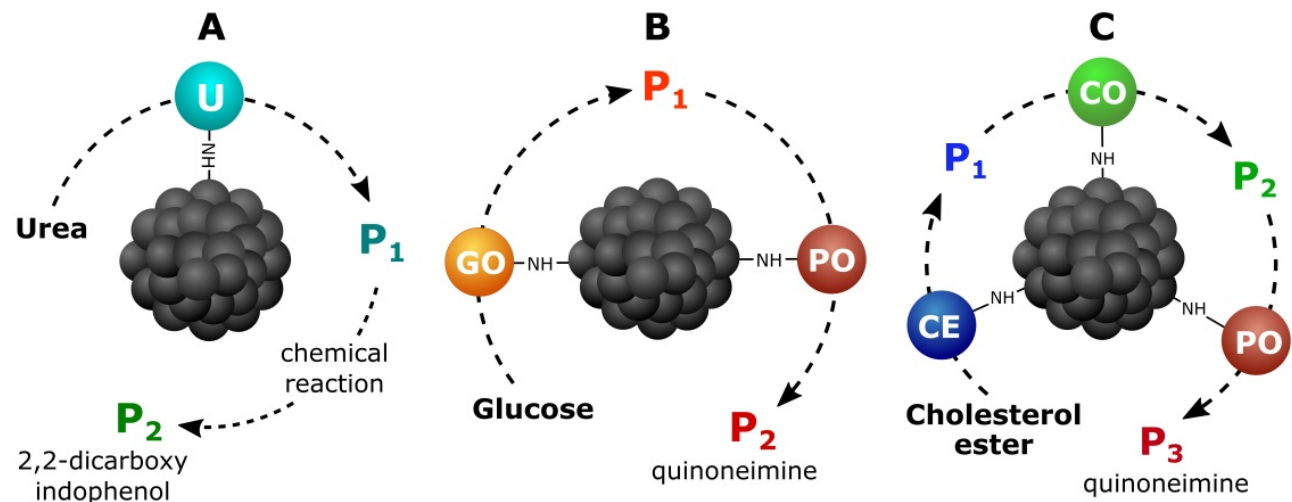

Figure 3. A schematic representation of working designed biochemical MND-enzymes test-systems. (A) Urea determination MND-monoenzyme complex: U- urease. (B) Glucose determination MND-bienzymes complex: GO glucose oxidase, PO-peroxidase. (C) Total cholesterol determination MND-three-enzymes complex: CE-cholesterol esterase, CO-cholesterol oxidase, PO-peroxidase.

At the first stage of the study it has been revealed that the weight ratio of the enzymes during covalent immobilization can be of importance in regards to the functional activity of designed MNDenzymes complexes. The MND-enzymes complexes showed the biggest activity and reaction product yields at the testing when for their fabrication the proteins mixture with an equal weight ratio of enzymes were used. When the amount of one of the enzymes was more or less than amount of the other ones in the protein mixture, the functional activities of obtained MND-enzymes complexes decreased about 2 times.

Calculations based on results of spectral analysis of the initial enzyme solutions and supernatants after immobilization suggested that at the experimental conditions $(\mathrm{pH}$ and molar values of buffer solution, amount of nanoparticles, temperature and duration of incubation), $1 \mathrm{mg}$ of MND bound $70 \mathrm{\mu g}$ of total amount of the enzymes at the creation of glucose determination system, $120 \mathrm{\mu g}$ of total amount of the enzymes at the creation of cholesterol determination system and $80 \mathrm{\mu g}$ of the enzyme at the creation of urea determination system.

In all cases studied, the covalently immobilized enzymes maintained functional activity and catalyzed the formation of the quinoneimine dye following the addition of corresponding analyte in the reaction mixture. In the control experiments without the analytes or when pristine MNDs were used, the colored product was not observed.

It was shown experimentally, that the developed test-systems were functional at a wide range of temperature. The enzymatic activity of MND-enzyme(s) complexes was evaluated by the yield of colored product while incubating the reaction solutions at $20-60{ }^{\circ} \mathrm{C}$ in a thermostat (TB- 85 Thermo Bath, Shimadzu, Japan). As it is seen on the graph (Fig. 4), the performance of the glucose biosensor was nearly invariant with temperature. Yet a slight increase of the yield of the colored product was detected in a temperature interval of $35-45{ }^{\circ} \mathrm{C}$. The activity of the cholesterol and urea test-systems displayed a pronounced temperature dependence. The maximum response of the cholesterol biosensor was observed at temperature close to $50{ }^{\circ} \mathrm{C}$ with fourfold increase of the reaction yield as compared to the room temperature $\left(20^{\circ} \mathrm{C}\right)$. The urea biosensor demonstrated the highest activity at a temperature interval of $40-50{ }^{\circ} \mathrm{C}$ resulting in $2-2.5$ times higher response than that at the room temperature. Obviously, the temperature effect on activity of the immobilized enzymes explains the observed fluctuation of biosensor performance. However, all test-systems were functioning quite effectively at the room temperature producing a quantity of the colored product sufficient to perform reliable spectral analysis. 


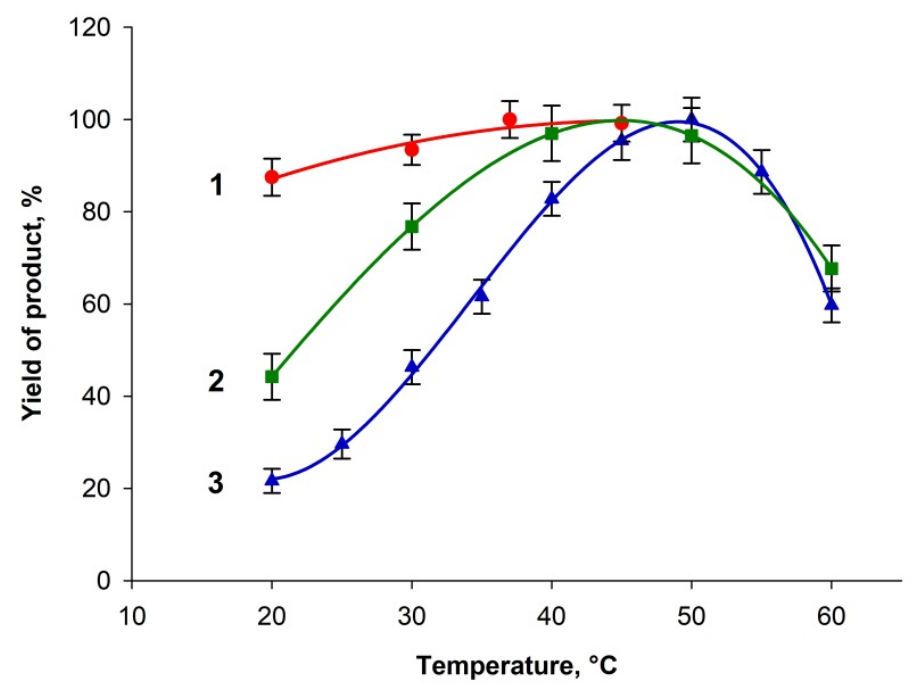

Figure 4. Dependence of product yield in reactions catalyzed by biochemical diagnostics MND-enzymes testsystems on temperature: 1-glucose determination test-system, 2-urea determination test-system, 3-total cholesterol determination system.

The effect of $\mathrm{pH}$ on the response of the developed test-systems was studied conducting experiments in a series of phosphate buffers $(10 \mathrm{mM})$ with $\mathrm{pH}$ values ranging from 5.0 to 9.0. As follows from the Fig. 4 , the glucose and cholesterol test-systems exhibited the maximal activity at slightly acidic to neutral conditions, while the urea test-system displayed an optimal response at neutral to slightly alkaline conditions. At the same time, all test-systems effectively function in DI water. The results of comparative study (Fig. 5) showed the almost identical response of MND-enzymes complexes to the target analyte in the neutral buffer $(\mathrm{pH} 7.0)$ and DI water. This can be considered as a certain advantage for their practical application.

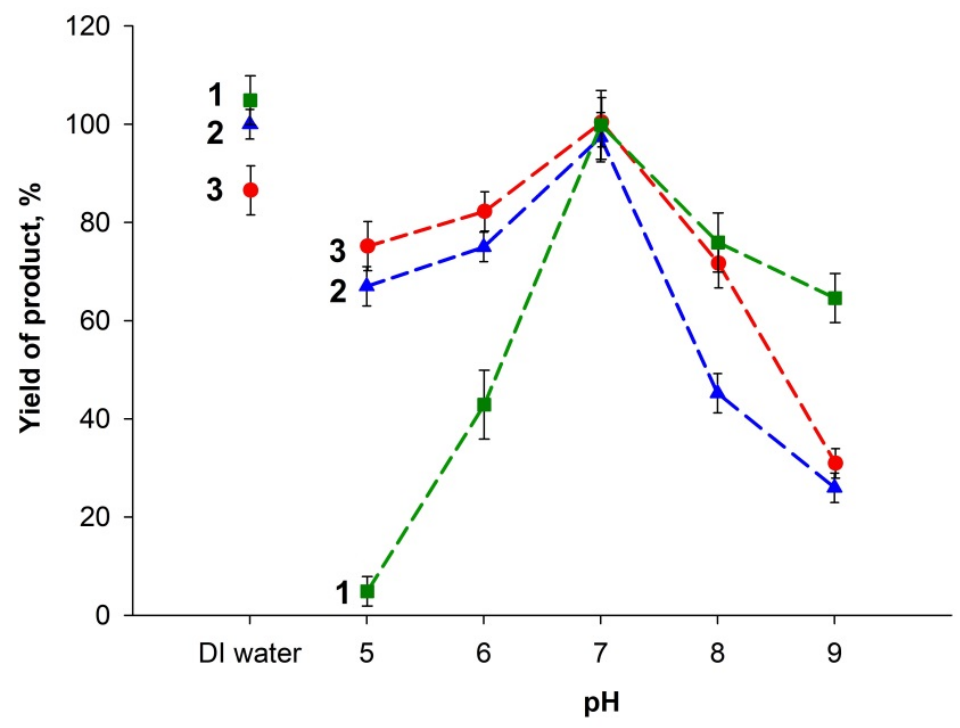

Figure 5. Dependence of yield of product in reactions catalyzed by biochemical diagnostics MND-enzymes testsystems on $\mathrm{pH}$ : 1-urea determination test-system, 2-total cholesterol determination test-system, 3-glucose determination system.

The influence of buffer composition on the response of glucose and cholesterol test-systems was investigated using different $20 \mathrm{mM}$ buffers with neutral $\mathrm{pH}$ (phosphate, ammonium acetate, bis-tris propane, sodium acetate and tris). The glucose test-system responded in all buffers almost the same as 
in DI water (Fig. 6). A slight decrease of the reaction rate with respect to DI water was observed only in the ammonium acetate and tris buffers (5-10\% and 10-15\%, correspondingly). The cholesterol testsystem was found to be sensitive to the buffer composition displaying a significantly lower response as compared with DI water measurements. The reduction was up to $30 \%$ in ammonium acetate, sodium acetate and tris buffers and reached $60 \%$ in bis-tris propane buffer (Fig. 6). The phosphate buffer was determined as the optimum medium for the both systems.

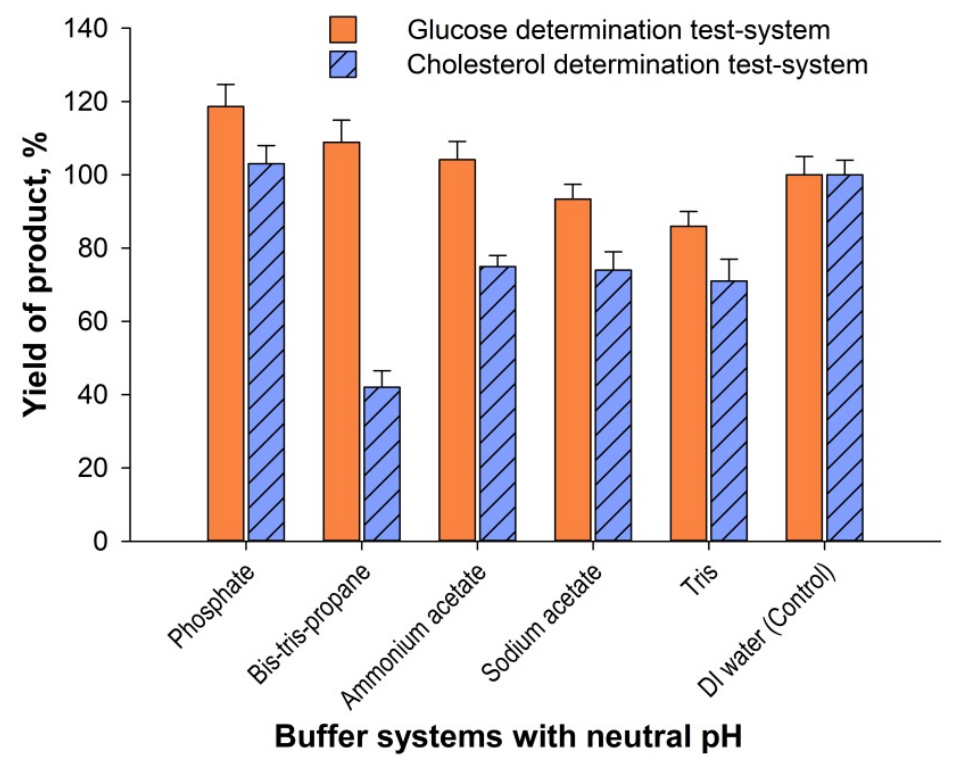

Figure 6. Formation of colored product in reactions catalyzed by glucose and cholesterol determination testsystems in different buffer systems $(20 \mathrm{mM}, \mathrm{pH}=7.0)$. The data are normalized for the product yield in DI water in measurements of glucose and cholesterol, respectively.

The fabricated test-systems were found to be stable when stored in the suspension form. The MNDenzymes complexes exhibited functional activity resulting in formation of the colored product in the presence of the corresponding analyte after storage in DI water at $4{ }^{\circ} \mathrm{C}$ for 3 months.
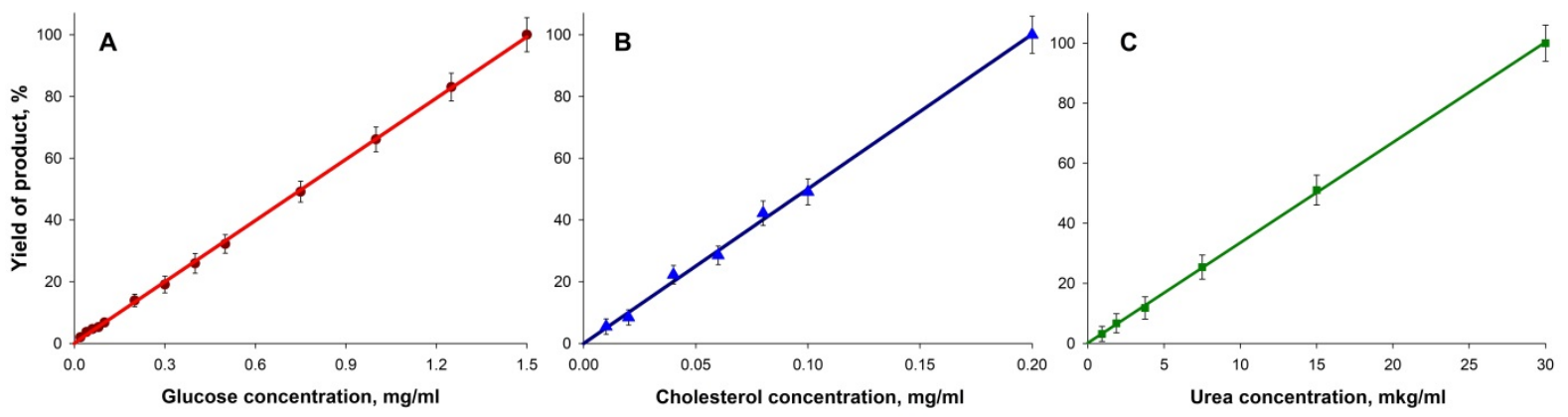

Figure 7. Formation of colored product in reactions catalyzed by MND-enzymes test-systems depending on concentration of suitable analyte: glucose (A), total cholesterol (B) and urea (C). The data are normalized for maximum values of product yield.

The experiments revealed that the test-systems demonstrated a linear output of the reaction product over a wide concentration range of all analytes (Fig. 7). The linear response was found for cholesterol, glucose and urea concentrations in the range of $0.01-0.20 \mathrm{mg} / \mathrm{ml}, 0.01-1.50 \mathrm{mg} / \mathrm{ml}$ and $0.9-30.0 \mu \mathrm{g} / \mathrm{ml}$, respectively. These results are essential in terms of developing sensing systems based on MND-enzymes complexes to determine the analyte concentration in biological liquids for clinical analysis. 


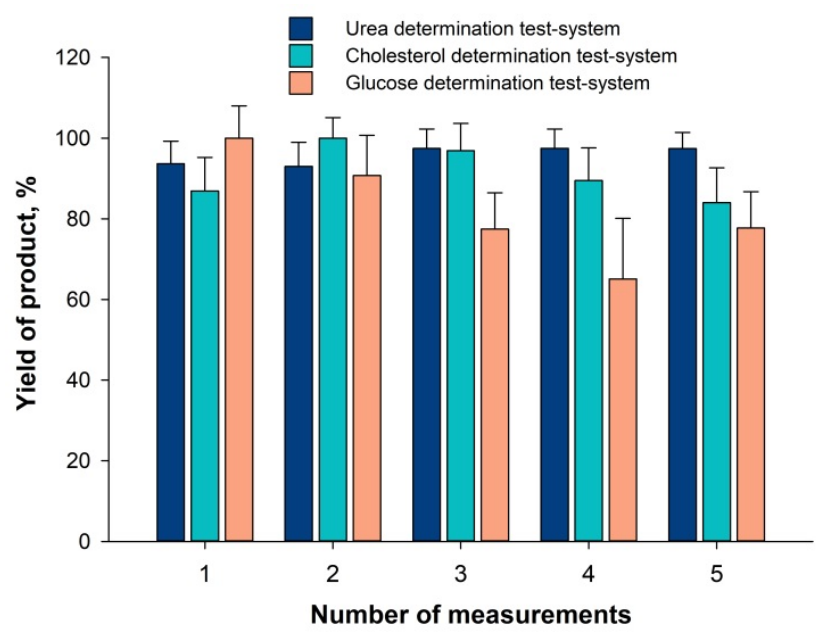

Figure 8. Formation of colored reaction product at multiple re-use of MND-enzymes test-systems. The data are normalized for maximum values of product yield.

The operational stability of the MND-enzymes complexes is illustrated in Fig. 8. As can be seen from the data, nearly the same rate of product formation was observed during repeated measurement of urea. This indicates that the immobilized enzymes were strongly bounded to the nanoparticles and not significantly inactivated by multiple testing of the MND-urease complex. From our point of view, dividing the detection of urea on enzymatic and nonenzymatic steps explains a high stability of this test-system as it allows avoiding a possible enzyme inactivation caused by chemical reagents (first of all, sodium hypochlorite and sodium hydroxide) during the nonenzymatic reaction. It was preliminary found that carrying out both reactions simultaneously leaded to a total loss of activity of the immobilized enzyme.
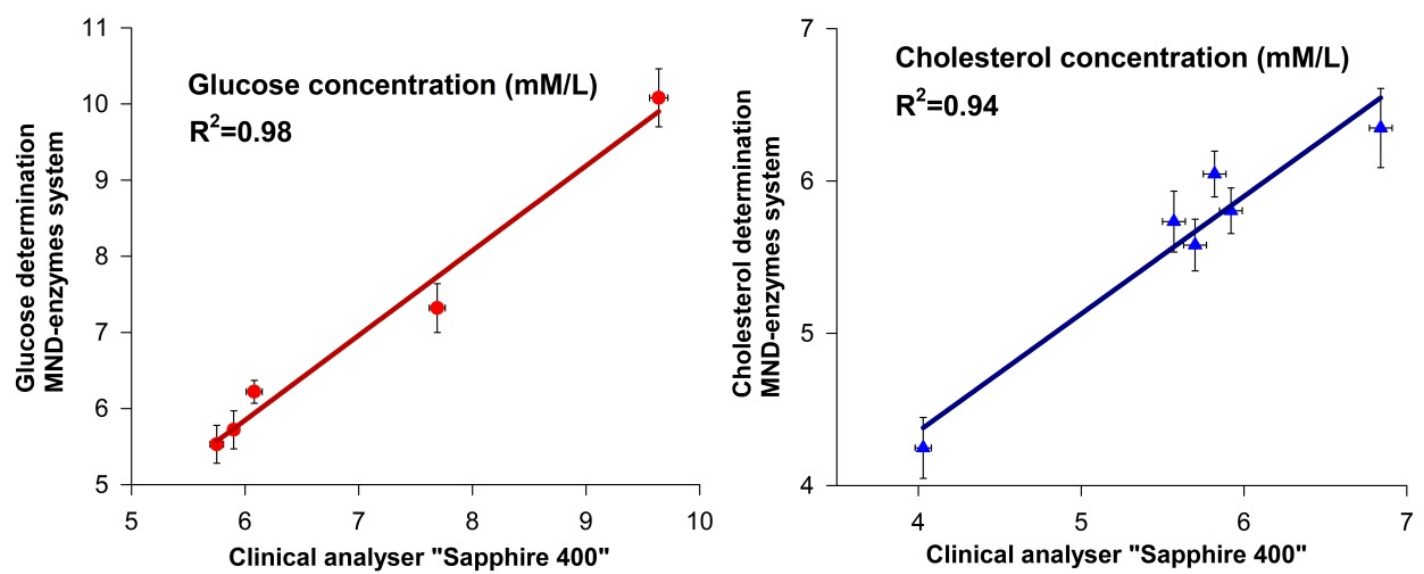

Figure 9. Correlation dependence of two measurement methods for glucose and cholesterol quantitation in human blood serum: via use of biochemical analyzer "Sapphire 400" and designed MND-enzymes systems.

Nearly the same rate of product formation was also observed during repeated measurement of cholesterol (Fig. 8). However, response of the MND-enzymes complex for glucose determination gradually decreased at the repeated testing of the analyte. The exact causes for reduced performance of this indicating complex are not clear yet and require further investigation.

Practical applicability of the designed testing systems was successfully demonstrated in comparative experiments on determination of glucose and cholesterol concentrations in human blood serum. Analysis of the analytes was performed on the same samples using MND-enzymes complexes and Sapphire 400 
biochemical analyzer as a reference tool. Quantitative estimations of glucose and cholesterol were obtained by comparing the yield of reaction product against the calibration curves (Fig. 7) when the MND-enzymes complexes were used. Good correlation was observed between results obtained with the test-systems and those with the analyzer (Fig. 9). The coefficients of determination $\left(\mathrm{R}^{2}\right)$ obtained from the analysis of glucose and cholesterol were of 0.98 and 0.94 , respectively.

\section{Conclusion}

Thus, the presented work demonstrates the applicability of modified detonation nanodiamonds as a carrier for creating systems for the biochemical diagnostics of physiologically important analytes. Testsystems for detection of urea, glucose and cholesterol were constructed by covalent immobilization of one (urease), two (glucose oxidase and peroxidase) or three (cholesterol esterase, cholesterol oxidase and peroxide) enzymes onto the benzoquinone functionalized surface of nanodiamonds. It was found that immobilized enzymes exhibited activity and catalyze the formation of colored reaction product upon addition of analytes. All MND-enzyme(s) complexes functioned in a wide range of temperatures and $\mathrm{pH}$, in DI water and buffers of different composition providing a linear yield of the reaction product in a wide range of analyte concentrations, exhibited activity after long-term storage in DI water at a temperature of $4{ }^{\circ} \mathrm{C}$. The MND-enzyme(s) complexes can be reused for the detection of the target analytes. The model biosensors are found to be suitable for repeated use and retain most of their activity after prolonged storage. Practical applicability of the developed test-systems is successfully demonstrated in experiments to estimate the glucose and cholesterol concentrations in human serum samples. We suggest that optimizing the conditions for constructing MND-enzyme(s) complexes (for example, fixing them on a solid substrate) will increase stability and efficiency of the test-systems in their multiple using.

Acknowledgments. This work was supported by the state budget allocated to the fundamental research at the Russian Academy of Sciences (project no. 0356-2016-0709).

\section{References}

1. T. R. Silva and I. C. Vieira, "Biosensor based on gold nanoparticles stabilized in poly (allyamine hydrochloride) and decorated with laccase for determination of dopamine," Analyst, vol. 141, no. 1, pp. 216-224, 2016.

2. C. Sun, L. Gao, D. Wang, M. Zhang, Y. Liu, Z. Geng, W. Xu, F. Liu, H. Bian, "Biocompatible polypyrrole-block copolymer-gold nanoparticles platform for determination of inosine monophosphate with bi-enzyme biosensor," Sensors and Actuators B: Chemical, vol. 230, pp. 521-527, 2016.

3. O. O. Soldatkin, I. S. Kucherenko, V. M. Pyeshkova, A. L. Kukla, N. Jaffrezic-Renault, A. V. Elskaya, S. V. Dzyadevych, A. P. Soldatkin, "Novel conductometric biosensor based on three-enzyme system for selective determination of heavy metal ions," Bioelectrochemistry, vol. 83, pp. 25-30, 2012.

4. Z. Li, C. Xie, J. Wang, A. Meng, F. Zhang, "Direct electrochemistry of cholesterol oxidase immobilized on chitosan-graphene and cholesterol sensing," Sensors and Actuators B: Chemical, vol. 208, pp. 505-511, 2015.

5. R. Mundaca-Uribe, F. Bustos-Ramirez, C. Zaror-Zaror, M. Aranda-Bustos, J. Neira-Hinojosa, C. Pena-Farfal, "Development of a bienzymatic amperometric biosensor to determine uric acid in human serum, based on mesoporous silica (MCM-41) for enzyme immobilization," Sensors and Actuators B: Chemical, vol. 195, pp. 5862,2014 .

6. L. Wang, X. Gao, L. Jin, Q. Wu, Z. Chen, X. Lin, "Amperometric glucose biosensor based on silver nanowires and glucose oxidase," Sensors and Actuators B: Chemical, vol. 176, pp. 9-14, 2013.

7. A. Boujakhrout, S. Jimenez-Falcao, P. Martinez-Ruiz, A. Sanchez, P. Diez, J.M. Pingarron, R. Villalonga, "Novel reduced graphene oxide-glycol chitosan nanohybrid for the assembly of amperometric enzyme biosensor for phenols," Analyst, vol. 141, pp. 4162-4169, 2016.

8. Z. Fan, Q. Lin, P. Gong, B. Liu, J. Wang, S. Yang, "A new enzymatic immobilization carrier based on graphene capsule for hydrogen peroxide biosensors," Electrochimica Acta, vol. 151, pp. 186-194, 2015. 
9. T. Gessei, T. Arakawa, H. Kudo, H. Saito, K. Mitsubayashi, "Amperometric biosensor based on enzyme immobilization with post process for medical and multiple application," Analytical Letters, vol. 47, pp. 1361-1374, 2014.

10. P. Raghu, T. M. Reddy, K. Reddaiah, B. E. K. Swamy, M. Sreedhar, "Acetylcholinesterase based biosensor for monitoring of malathion and acephate in food samples: A voltammetric study," Food Chemistry, vol. 142, pp. 188-196, 2014.

11. I. M. Apetrei and C. Apetrei, "Amperometric biosensor based on polypyrrole and tyrosinase for the detection of tyramine in food samples," Sensors and Actuators B: Chemical, vol. 178, pp. 40-46, 2013.

12. D. Ling, N. Lee, T. Hyeon, "Chemical synthesis and assembly of uniformly sized iron oxide nanoparticles for medical applications," Accounts of Chemical Research, vol. 48, no. 5, pp. 1276-1285, 2015.

13. S. M. Davachi and B. Kaffashi, "Preparation and characterization of poly L-Lactide/Triclosan nanoparticles for specific antibacterial and medical application," International Journal of Polymeric Materials and Polymeric Biomaterials, vol. 64, no. 10, pp. 497-508, 2015.

14. Y. Wang, Q. Zhao, N. Han, L. Bai, J. Li, J. Liu, E. Che, L. Hu, Q. Zhang, T. Jiang, S. Wang, "Mesoporous silica nanoparticles in drug delivery and biomedical applications," Nanomedicine: Nanotechnology, Biology and Medicine, vol. 11, no. 2, pp. 313-327, 2015.

15. A. Gismondi, G. Reina, S. Orlanducci, F. Mizzoni, S. Gay, M. L. Terranova, A. Canini, "Nanodiamonds coupled with plant bioactive metabolites: A nanotech approach for cancer therapy," Biomaterials, vol. 38, pp. 22-35, 2015.

16. Y. Yang, A. M. Asiri, Z. Tang, D. Du, Y. Lin, "Graphene based materials for biomedical applications," Materials Today, vol. 16, no. 10, pp. 365-373, 2013.

17. L. Lai and A. S. Barnard, "Functionalized nanodiamonds for biological and medical applications," Journal of Nanoscience and Nanotechnology, vol. 15, no. 2, pp. 989-999, 2015.

18. G. Yesiller and M. K. Sezginturk, "A new methodology for the determination of enzyme activity based on carbon nanotubes and glucose oxidase," Journal of Pharmaceutical and Biomedical Analysis, vol. 115, pp. 254259, 2015.

19. V. N. Mochalin, O. Shenderova, D. Ho, Y. Gogotsi, "The properties and applications of nanodiamonds," Nature Nanotechnology, vol. 7, pp. 11-23, 2012.

20. A. Krueger, "New carbon materials: biological applications of functionalized nanodiamond materials," Chemistry - A European Journal, vol. 14, pp. 1382-1390, 2008.

21. A. M. Schrand, S. A. C. Hens, O. A. Shenderova, "Nanodiamond particles: properties and perspectives for bioapplications," Critical Reviews in Solid State and Materials Sciences, vol. 34, pp. 18-74, 2009.

22. J. M. Say, C. van Vreden, D. J. Reilly, L. J. Brown, J. R. Rabeau, N. J. C. King, "Luminescent nanodiamonds for biomedical applications," Biophysical Reviews, vol. 3, no. 4, pp. 171-184, 2011.

23. D. Ho, C. H. K. Wang, E. K. H. Chow, "Nanodiamonds: The intersection of nanotechnology, drug development, and personalized medicine," Science Advances, vol. 1, no. 7, pp. 1-14, 2015.

24. V. S. Bondar and A. P. Puzyr, "Nanodiamonds for Biological Investigations," Physics of the Solid State, vol. 46, no. 4, pp. 716-719, 2004.

25. A. P. Puzyr, A. V. Baron, K. V. Purtov, E. V. Bortnikov, N. N. Skobelev, O. A. Mogilnaya, V. S. Bondar, "Nanodiamonds with novel properties: A biological study," Diamond and Related Materials, vol. 16, no. 12, pp. 2124-2128, 2007.

26. A. P. Puzyr, V. S. Bondar, A. A. Bukayemsky, G. E. Selyutin, V. F. Kargin, "Physical and chemical properties of modified nanodiamonds," NATO Science Series II: Mathematics, Physics and Chemistry, vol. 192, pp. 261$270,2005$.

27. N. Gibson, O. Shenderova, T. J. M. Luo, S. Moseenkov, V. Bondar, A. Puzyr, K. Purtov, Z. Fitzgerald, D. W. Brenner, "Colloidal stability of modified nanodiamond particles," Diamond and Related Materials, vol. 18, pp. 620-626, 2009.

28. A. P. Puzyr and V. S. Bondar, "Method of Production of Nanodiamonds of Explosive Synthesis with an Increased Colloidal Stability," RU Patent No. 2252192, 2005.

29. J. Brandt, L. O. Andersson, J. Porath, "Covalent attachment of proteins to polysaccharide carriers by means of benzoquinone," Biochimica et Biophysica Acta, vol. 386, pp. 196-202, 1975. 
30. M. A. Mateescu, G. Weltrowska, E. Agostinelli, R. Saint-Andre, M. Weltrowski, B. Mondovi, "Ready to use pbenzoquinone-activated supports for biochemical coupling, with special applications for laccase immobilization," Biotechnology Techniques, vol. 3, pp. 415-420, 1989.

31. L. A. Osterman, Chromatography of Proteins and Nucleic Acids. Moscow: Nauka, 1985.

32. A. N. Yeremin, T. V. Semashko, R. V. Mikhailova, "Cooxidation of phenol and 4-aminoantipyrin, catalyzed by polymers and copolymers of horseradish root peroxidase and Penicillium funiculosum 46.1 glucose oxidase," Applied Microbiology and Biotechnology, vol. 42, no. 4, pp. 399-408, 2006.

33. F. G. Menezes, A. C. O. Neves, D. F. Lima, S. D. Lourenco, L. C. Silva, K. M. G. Lima, "Bioorganic concepts involved in the determination of glucose, cholesterol and triglycerides in plasma using the enzymatic colorimetric method," Química Nova, vol. 38, no.4, pp. 588-594, 2015.

34. A. Tabacco, F. Meiattini, E. Moda, P. Tarli, "Simplified enzymic/colorimetric serum urea nitrogen determination," Clinical Chemistry, vol. 25, no. 2, pp. 336-337, 1979.

35. R. L. Searcy, J. E. Reardon, J. A. Foreman, "A new photometric method for serum urea nitrogen determination," The American Journal of Medical Technology, vol. 33, no. 1, pp. 15-20, 1967. 